\title{
Establish New Supporting Point of Effective Teaching for Weak Schools with Teaching and Studying Plan
}

\author{
Guijun Ma \\ College of Educational Science, Ningxia Normal University, Guyuan Ningxia, 756000, China
}

Keywords: Teaching and studying plan, Weak schools, Effective teaching, New supporting point.

\begin{abstract}
In terms of current teaching status of Chinese weak schools, there are many unavoidable problems such as low student quality, backward teaching method and low efficiency of classroom teaching. How to establish new supporting point of effective teaching for weak schools becomes one of the key research directions in the current educational world. Due to long-term influence of traditional teaching mode and concept on Chinese educational world, the efficiency of classroom teaching in each stage and teaching achievements of each educational institution are not optimistic. Teaching in weak schools is always a major concern of the state and the society. Some educational expert has pointed out that reasonable use of teaching and studying plan mode can improve the teaching status of weak schools effectively.
\end{abstract}

\section{Introduction}

Teaching and studying plan integrates teachers' teaching plan and students' studying plan. Teaching with teaching and studying plan can promote good interaction between teachers and students in class and improve classroom learning efficiency of students effectively [1-2].Teaching and studying plan is the most important basis for teachers' teaching and implementation of teaching activities. It mainly covers disciplinary subject, class hour, class type and teaching method, objective, content and activity allocation [3].To change the teaching status of Chinese weak schools effectively and further promote the development of Chinese educational business, this paper discusses the establishment of new supporting point of effective teaching for weak schools with teaching and studying plan as below.

\section{Unavoidable teaching difficulties in Chinese weak schools}

From the perspective of comprehensive analysis, there are various factors causing weak schools, among which student quality is the major influencing factor. The source of students of an educational institution is greatly linked to historical reasons of its own development. However, student distribution policy of relevant administrative department of local education is the primary decisive factor. Under the influence of these primary factors, students in such schools do not have much difference in both quantity and quality. Therefore, a certain vicious circle forms and the bad situation of weak schools cannot be alleviated and improved effectively.

From the perspective of reality, in the process of classroom teaching in many weak schools, though teachers are eloquent on the platform, few students can really listen to them. Most students live in their own "small world [4-5]" in class. They talk, read extracurricular books and sleep.... In such a situation, many teachers are unwilling to maintain classroom discipline. They choose to continue their teaching and even think that reminding such students will disturb the subsequent course teaching. Therefore, classroom is undoubtedly a suffering for both teachers and students in such a situation.

Due to long-term influence of traditional teaching concept and mode, “duck-stuffing” type of teaching [6-7] can be seen everywhere in actual classroom teaching process. For schools with weak 
source of students, such teaching mode and method cannot apply to student group or meet students' learning demands. Therefore, lesson preparation, preparation before class and classroom teaching activities of teachers almost become idle work. Therefore, in such a situation, no matter how teachers prepare lesson, give lesson and correct homework in daily teaching work, the teaching effect is insignificant if students cannot accept it.

According to the current development status of Chinese educational world, the reality of poor student quality cannot be changed effectively for the time being. To change this bad situation effectively, the educational world must start with weak schools and aim at transforming their teaching concept, management concept, teachers' teaching method and students' learning style effectively.

\section{Attempt to use teaching and studying plan for teaching}

In recent years, integrated teaching and studying plan has gradually emerged and become an effective means to standardize teaching behaviors of teachers and adjust learning behavior of students.

(1) Lead teachers in weak schools to the establishment of scientific educational concepts of "accepting differences, respecting personality, teaching students according to their ability and teaching through lively activities [10]".In actual teaching process, teachers in weak school should fully consider actual learning situation of students, "encourage progress and be progressive" in the real sense and lead students to "active and harmonious development" in the learning process [11-12].Meanwhile, teachers should also be good at discovering and exploring highlights of each student in teaching, carry forward their highlights, give them appropriate inspiration and guide them to make progress slowly in learning. In addition, teachers should also think from the perspective of students, get along with them with tolerant and comprehensive attitudes and help them grow healthily.

(2) Improve collective lesson preparation with lesson preparation group as basic unit, optimize lesson preparation mode constantly and improve effective lesson preparation. Teachers in weak schools should start from teaching reality based on the principle of "teaching by learning", further promote secondary development of Chinese teaching courses and the development of exercise preparation based on comprehensive learning status of students, implement lesson preparation policy from individual lesson preparation - collective lesson preparation - advance course experiment collective secondary discussion - unified framework - individuation improvement in teaching preparation process based on "unit" and meanwhile follow lesson preparation and teaching criteria of "teaching according to their ability and layered teaching" so as to maximize the function of personalized and layered teaching and studying plan in actual teaching process. Meanwhile, teachers should also fully consider realistic features of students in weak schools such as poor ability of independent study and unscientific learning method and habits, harmonize teaching and studying plan and try to realize "one course, one plan; one plan, one exercise [15]" so as to guarantee effective implementation of teaching and studying plan in form and improve the efficiency of teachers' teaching and students' learning constantly.

(3) Cancel various exercise books and guidance materials as much as possible and improve the preparation of school-based exercises of weak schools effectively. In the process of school-based exercise preparation, weak schools should make preparation with lesson preparation group as unit and implement group leader responsibility system. A number of educational researchers have pointed out that weak schools can prepare school-based exercises with the following mode: individual preparation - collective discussion - preparation of test questions - unified framework - submission for examination - improvement [16].From comprehensive perspective, the preparation of exercises can be divided into three types, namely selected preparation, adaptation and self-preparation [17].It is worth noting that exercises prepared by teachers shall not deviate from teaching and studying plan; instead, they should be unified with teaching and studying plan so that teachers can integrate them into actual teaching process better. That is to say, exercises must have corresponding flexibility. 
(4) Further enhance the construction of efficient classroom. In this aspect, teachers in weak schools can start with the improvement of students' learning interest, make arrangement for students' independent intervention in classroom leading-in link through mutual questioning and allow students to experience the joy of participating in learning to the greatest extent and improve their learning enthusiasm and confidence; aim at simplifying contents, reduce the difficulty of teaching contents appropriately and teach briefly and succinctly so that students can master contents of teaching materials step by step. Under the guidance of "learning plan and learning guidance plan [18]", weak schools must take corresponding measures to reduce and even abolish all purchased guidance materials arranged for students, reduce the quantity of homework of students appropriately so as to reduce their learning pressure, prepare exercises and homework pertinently, try to realize "homework doing, correcting and feedback” in the real sense and allow students to learn easily.

\section{Supporting point function of implementing integrated teaching and studying plan for teaching in weak schools}

Scientific integrated teaching and studying plan is an effective means to standardize teachers' teaching behavior and regulating students' learning behavior.

Establish a visible learning supporting point for students undisciplined in learning and further promote the implementation of teaching contents.

Create a supporting point of interesting teaching situation for boring teaching atmosphere and further promote students' learning enthusiasm and interest.

Establish a supporting point and platform of effective interaction for teaching classroom under traditional mode and further enhance the interaction between teachers and students in study and life.

Establish a new teaching supporting point of "first self-study and then key teaching” for teachers and students effectively and further stimulate students' awareness of independent study and inquiry effectively.

Establish an effective supporting point for students to realize classroom practice and timely feedback and further promote students' repeated cognition and consolidation of the knowledge learnt and help them develop good learning habits.

\section{Systematic overall teaching implementation process of teaching and studying plan}

\section{Prepare teaching and studying plan scientifically with collective wisdom and power}

During the preparation of teaching and studying plan, everyone in the lesson preparation group should participate, cooperate, discuss and follow the procedure seriously. Basic procedure: division of work in advance, personal "preparation”, collective "discussion[19]", lesson preparation by major teachers, collective improvement of final draft and common discussion, modification and finalization. Each teaching and studying plan centralizes collective wisdom of lesson preparation group.

\section{Implant teaching and studying plan into the teaching mode of "first learning and then teaching"}

First, teachers in weak schools should constantly stimulate the confidence and enthusiasm of students poor in study and create more learning opportunities for them appropriately;

Second, guide students to specify and concretize their self-study requirements, such as the scope of reading, problems of thinking and experimental contents or practice to be completed, so that students can study step by step rather than study blindly without any learning effect.

Third, be good at using positive educational function of appreciation and encouragement skillfully so as to allow students to set up learning confidence constantly in the learning process, further arouse their interest in learning and help them develop scientific learning habits.

"First learning" means that teachers guide students in independent study scientifically. Teachers in weak schools can give full play to teaching and studying plan which is a good mode, establish an 
effective platform for "first learning" for students and create and provide more independent learning opportunities for them. It is worth noting that contents and difficulty of teaching and studying plan must conform to the learning reality of students. Attention should be paid to avoid excessive classroom contents and excessively difficult teaching contents which will boost learning pressure of students invisibly. "Then teaching" means that teachers in weak schools further guide students in group discussion about problems in "cooperative research" through "interactive exchange among students" or "interactive exchange between teachers and students"[20] on the basis of guiding them in independent study so that students can master teaching contents and complete teaching objectives easily in good learning atmosphere with mutual help. In the whole process of "first learning" and "then teaching", leaders of teachers in weak schools always play a role as leader and instructor who mainly make comprehensive explanations for students when discovering individual special problems or common problems.

\section{Acknowledgement}

Issue: scientific research project of Ningxia Normal University in 2017, study on innovation of classroom teaching mode in Guyuan No.6 Middle School NXSFYB1772.

\section{Conclusion}

In conclusion, for the construction of new teaching supporting point of weak schools, it is required to conduct software and hardware construction pertinently based on full self-diagnosis, especially transform educational teaching concept, strengthen construction in school management, teaching staff construction, student education, school-running conditions and educational environment and make schools take a featured way of leaping development.

\section{References}

[1] Bao Chuanyou. Lack of Confidence and Trust Reestablishment of Rural Weak Schools, Chinese Journal of Education, 2017, (03): 50-53.

[2] Fan Gaixia, Chen Zupeng. Study on Construction Path of Chinese Rural Weak Schools from Multi-dimensional Perspective, Educational Exploration, 2016, (03): 45-51.

[3] Lou Lizhi, Liu Wenwen. Loss of Backbone Teachers in Rural Weak Schools and Reply, Teacher Education Research, 2016, 28 (02): 75-80.

[4] Tang Zhenhua, Xia Xueyu. Establish Schools with Spirit and Explore Way with Science Interpretation on Transformation of Suburban Weak School No.7 Primary School of Bazhou District, Educational Science Forum, 2017, (03): 71-76.

[5] Deng Liang. Study on Transformation of Weak Schools from the Perspective of School Culture Take Middle School A for Example, Elementary Education Studies, 2016 (01): 24-27.

[6] Mo Lijuan. “Corruption” and "Escape”: Obedience and Defiance of Teachers in Rural Weak Schools under Pressure of Examination, Contemporary Educational Science, 2017, (01): 62-67.

[7] Zhou Teng. Rethink on Practice of Value Reconstruction of Students in Weak Schools, Tibetan Education, 2017, (01): 36-39.

[8] Long Run, Zhong Pengli. "Reasons for Difficulty" and "Path of Action" for Transformation of Rural Weak Schools, Modern Education Sciences, 2016, (06): 16-36.

[9] Xiong Zongqiang, Hong Shilin, Zhang Shilei. Exploration on Promotion of Development of Urban Weak Schools by Informatization Means, Educational Teaching Forum, 2016, (52): 257-259. 
[10]Chen Fabao, Yang Xiaojie, Xie Lili. Study on Status of Teachers' Professional Development in Weak Schools under U-S Cooperation Background - Take a Rural Middle School in Zhejiang for Example, Journal of Educational Development, 2016, (11): 62-65.

[11]Zhou Yu, Zhang Hongxia, Wang Jiamin. Exploration on Localization of Open Educational Resources - Take Application of Mooc in Rural Weak Schools for Example, Education and Teaching Study, 2016, 30 (10): 29-35.

[12] Shan Dasheng. Progress of Transformation Plan of Weak Schools of Compulsory Education in Rural Areas and Rural Education Development Prospect, Journal of Yan'an University (Social Science Edition), 2016, 38 (05): 109-125.

[13]Zhu Hong. Discussion on Strategies for Effective Review of Mathematics for College Entrance Examination in Schools with Weak Foundation of Students, China Training, 2016, (10): 155-157.

[14]Dong Meiying. Analysis on Path of Obtaining Identity of "Elite School" and Discussion on Transformation of Weak Schools - from the Perspective of Capital, Education Research Monthly, 2016, (09): 47-52.

[15]Xiong Zongqiang, Hong Shilin, Zhang Shilei. Exploration on Promotion of Development Mechanism of Urban Weak Schools by Informatization Means - Based on the Perspective of Feasible Capability Theory, Modernization of Education, 2016, (18): 48-50.

[16] Yang Jifu, Huang Xiaona. Several Suggestions on Reconstruction of Teacher Culture in Weak Schools in Urbanization Process, Journal of Mudanjiang Institute of Education, 2016, (07): 44-45.

[17]Chen Junke, Ma Jiaoyang. Inspiration of Teacher Retention Measure in American Weak Schools on China, Journal of Henan Institute of Technology, 2016, 36(06): 78-81.

[18]Shan Qianqian. Problems Existing in English Disciplinary Construction of Rural Weak Schools of Compulsory Education and Countermeasures, Journal of Shandong Agriculture and Engineering University, 2016, 33(06): 138-139.

[19]Zhang Jin. Study on Cases of Transformation of Rural Weak Schools in Gansu Based on SWOT Analysis Method, Educational Teaching Forum, 2016, (17): 19-20.

[20]Hong Yulong, Du Huiqi. Pave an Appropriate Way for Rising of Weak Schools, People's Education, 2016, (08): 41-43. 